\title{
(In)visibilidades da saúde da população LGBT no Programa de Pesquisa para o SUS (PPSUS), Brasil
}

\author{
(In)visibility of the health concerning the LGBT population in the Research \\ Program for the SUS (PPSUS), Brazil
}

\section{(In)visibilidades de la salud de la población LGBT en el Programa de Investigación para el SUS (PPSUS), Brasil}

\author{
Ana Cristina de Souza Mandarino ${ }^{1,2, a}$ \\ anamandarino@gmail.com | https://orcid.org/oooo-0002-1628-7363 \\ Jhonatan da Silva Queirós ${ }^{3, b}$ \\ jhon.queiros@gmail.com | https://orcid.org/oooo-0003-4673-305 \\ Wilson Couto Borges ${ }^{4, c}$ \\ wcborges1@yahoo.com.br | https://orcid.org/o0oo-0002-2785-3658 \\ Estélio Gomberg ${ }^{3, d}$ \\ estelio68@gmail.com | https://orcid.org/oooo-0003-3603-1872 \\ ${ }^{1}$ Universidade Federal de Sergipe. São Cristóvão, SE, Brasil. \\ ${ }^{2}$ Centro de Estudos Internacionais, Instituto Universitário de Lisboa. Lisboa, Portugal. \\ ${ }^{3}$ Universidade Estadual de Santa Cruz. Ilhéus, BA, Brasil. \\ ${ }^{4}$ Fundação Oswaldo Cruz, Instituto de Comunicação e Informação Científica e Tecnológica em Saúde, Programa de \\ Pós-Graduação em Informação e Comunicação em Saúde. Rio de Janeiro, RJ, Brasil. \\ a Doutorado em Comunicação e Cultura pela Universidade Federal do Rio de Janeiro. \\ ${ }^{\mathrm{b}}$ Graduando em História pela Universidade Estadual de Santa Cruz. \\ c Doutorado em Comunicação pela Universidade Federal Fluminense. \\ d Doutorado em Saúde Pública pela Universidade Federal da Bahia.
}

\section{Resumo}

Uma das importantes conquistas para consolidação da democracia no Brasil foi a criação e a efetivação da Lei de Acesso à Informação (LAI). Neste artigo, interessa relacionar a LAI, a institucionalização da Política Nacional de Informação e Informática em Saúde e o Programa de Pesquisa para o SUS (PPSUS), privilegiando as Fundações de Amparo à Pesquisa (FAP), um dos atores deste Programa do Ministério da Saúde (MS). O método utilizado para o estudo foi a realização de um levantamento de editais publicados e de projetos de pesquisa contemplados no PPSUS, voltados para a instituição da Política Nacional de Saúde Integral de Lésbicas, Gays, Bissexuais, Travestis e Transexuais (PNSILGBT). Ao observar, nos sites das instituições mencionadas, os registros de projetos de pesquisa relacionados à saúde da população LGBT, valorizou-se a dimensão da usabilidade, apreendendo desorganização nas apresentações dos editais e dos resultados, o que proporciona ineficiência na sua operacionalização e na funcionalidade das formulações de políticas no campo da saúde.

Palavras-chave: Lei de Acesso à Informação; Informação em saúde; Política de pesquisa em saúde; Internet; Saúde da população LGBT. 


\begin{abstract}
One of the important achievements for consolidation of the democracy in Brazil was the LAI - Lei de Acesso à Informação (access to information law) passed by Brazilian government and its implementation. In this article, we intend to examine how the LAI, the institutionalization of the Política Nacional de Informação e Informática em Saúde (a national policy on health information) and the PPSUS - Programa de Pesquisa para o SUS (research program for the SUS) are related, favouring the FAP - Fundações de Amparo à Pesquisa (foundations for research support), one of the actors of this program developed by Ministry of Health of Brazil. A survey of published call for proposals and research projects selected by the PPSUS aiming the institution of the PNSILGBT - Política Nacional de Saúde Integral de Lésbicas, Gays, Bissexuais, Travestis e Transexuais (national integral health policy for lesbians, gays, bisexuals, transvestites and transsexuals) was carried out. Observing the research registers about the health of the LGBT population on sites of mentioned institutions, from the perspective of the usability dimension, we identified a disorganization in the presentations of the call for proposals and their result, providing inefficiency in their viability and in the performance of the policy formulation in health field aiming the LGBT population.
\end{abstract}

Keywords: Access to information law; Health information; Health research policy; Internet; Health of the LGBT population.

\title{
Resumen
}

Una de las importantes conquistas para la consolidación de la democracia brasileña fue la creación y la realización de la LAI - Lei de Acesso à Informação (ley de acceso a la información). En este artículo buscamos relacionar la LAI, la institucionalización de la Política Nacional de Informação e Informática em Saúde (política nacional de información en salud) y el PPSUS - Programa de Pesquisa para o SUS (programa de investigación para el SUS), privilegiando las FAP - Fundações de Amparo à Pesquisa (fundaciones de auxilio a la investigación), uno de los actores de este programa del Ministerio de Salud de Brasil. El método utilizado para el estudio apresentado aquí ha sido un levantamiento de llamadas públicas y de proyectos de investigación seleccionados en el PPSUS, destinados a la institución de la PNSILGBT - Política Nacional de Saúde Integral de Lésbicas, Gays, Bissexuais, Travestis e Transexuais (política nacional de salud integral de lesbianas, gays, bisexuales, travestis y transexuales. Al observar, en lo portal de las instituciones mencionadas, registros de proyectos de investigación relacionados con la salud de la población LGBT, del punto de vista de la dimensión de la usabilidad, concluimos que hay desorganización en las presentaciones de las llamadas públicas y de los resultados, lo que proporciona ineficiencia en su realización y en la funcionalidad de las formulaciones de políticas del gobierno en el campo de la salud.

Palabras clave: Ley de acceso a la información; Información en salud; Política de investigación en salud; Internet; Salud de la población LGBT.

Este artigo faz parte do dossiê $\mathbf{4 0}$ anos do movimento LGBT: visibilidades e representações.

Contribuição dos autores: os autores conceberam o artigo com atividades equitativas na sua elaboração.

Declaração de conflito de interesses: não há.

Fontes de financiamento: Edital Universal do Conselho Nacional de Desenvolvimento Científico e Tecnológico (CNPq) 2016.

Considerações éticas: não há.

Agradecimentos/Contribuições adicionais: não há.

Histórico do artigo: submetido: 07 fev. 2019 | aceito: 22 ago. 2019 | publicado: 12 set. 2019.

Apresentação anterior: não houve.

Licença CC BY-NC atribuição não comercial. Com essa licença é permitido acessar, baixar (download), copiar, imprimir, compartilhar, reutilizar e distribuir os artigos, desde que para uso não comercial e com a citação da fonte, conferindo os devidos créditos de autoria e menção à Reciis. Nesses casos, nenhuma permissão é necessária por parte dos autores ou dos editores. 


\section{Introdução}

Uma das importantes conquistas para consolidação da democracia em nosso país foi a criação e a efetivação da Lei de Acesso à Informação (LAI). Não sem avanços e rupturas, a LAI representou um movimento na direção da melhoria da qualidade de produção, armazenamento e disponibilização de informações que permitem maior transparência ao Estado, mas igualmente a possibilidade de que uma gestão mais efetiva desses dados possa fomentar o crescimento do campo da Ciência, Tecnologia \& Invovação (CT\&I). Ainda que esse fenômeno possa ser observado em todas as áreas, nosso foco neste artigo é a relação entre a LAI e o fomento promovido pelo Programa de Pesquisa para SUS (Sistema Único de Saúde), tendo em vista o impacto que exerce sobre a vida de todos os brasileiros.

Diante desse cenário, nos interessa neste artigo apresentar e, em alguma medida, relacionar a LAI, a institucionalização da Política Nacional de Informação e Informática em Saúde e o Programa de Pesquisa para o SUS, tendo como horizonte o aumento da capacidade de tais pesquisas aumentarem a possibilidade de tomadas de decisões mais efetivas (com base em evidências empíricas) com o consequente aumento de benefício e redução de custos para a população. Nesse processo, com vistas a qualificar ainda melhor a gestão em saúde no Brasil, emergem como atores centrais as Fundações Públicas de Amparo à Pesquisa (FAPs) de modo a, num processo descentralizado, contribuir para a melhoria da pesquisa em saúde em todo o país. Com esta perspectiva, colocamos luz sobre os editais vinculados ao Programa de Pesquisa para o SUS como forma de mapear as possíveis conexões entre ele e a Agenda Nacional de Prioridades de Pesquisa em Saúde (ANPPS).

De um quadro geral das necessidades de pesquisa em saúde, nossa atenção neste artigo se volta para as investigações que envolvem a população de lésbicas, gays, bissexuais e transgêneros (LGBT), inscritas numa dinâmica em que políticas públicas específicas se fazem necessárias. Para tanto, observadas as lutas travadas por esse grupo desde os anos 1980, julgamos ser central o debate em torno da relação gênero e saúde, face à presença histórica de processos de sua discriminação e exclusão na formação social brasileira. Nos termos aqui colocados, ganha relevância e densidade a observância da Política Nacional de Saúde Integral de Lésbicas, Gays, Bissexuais, Travestis e Transexuais com vistas ao processo mais amplo de compreensão e respeito aos direitos humanos, especialmente a partir da perspectiva de uma saúde integral, universal e equânime.

É imprescindível às informações de instituições públicas, a organização, a preservação de fontes e os suportes documentais, bem como a gestão estratégica para a facilitação do acesso a elas pelos cidadãos, com transparência e objetividade para sua fácil compreensão, reportando aos princípios da administração pública, da inviolabilidade da vida privada e da intimidade ${ }^{1-3}$. Assim, é possível contribuir também para a melhoria e automação dos processos de gestão da organização pública. Com o objetivo de proporcionar uma maior efetividade na prestação de serviços, foi criado pelo governo brasileiro um canal central, como pontuam Barbosa e outros": "O portal governamental é um canal único na rede mundial onde o cidadão pode obter informações e solicitar serviços aos mais diferentes agentes governamentais de uma maneira simples e interativa."

Nesse sentido, os órgãos administrativos públicos devem assegurar às pessoas físicas e jurídicas o direito de acesso à informação, como dispõe o art. $2^{\circ}$ da Resolução CNMP No 89/2012) e garantir uma gestão transparente da informação, propiciando amplo acesso à informação e sua divulgação de forma integrativa.

O acesso à informação é uma norma positivada, com previsão no inciso XXXIII do caput do art. $5^{\circ}$, no inciso II do $\S 3^{\circ}$ do art. 37 e no $\S 2^{\circ}$ do art. 216 da Constituição Federal da República do Brasil de $1988^{5}$.

Além disso, a Lei de Acesso à Informação (Lei n. ${ }^{0}$ 12.527, de 18 de novembro de 2011) é aplicada por disposição expressa de seu art. $1^{\circ}$, parágrafo único, inciso I: “Art. $1^{\circ}$ Esta Lei dispõe sobre os procedimentos a serem observados pela União, Estados, Distrito Federal e Municípios, com o fim de garantir o acesso a informações previsto no inciso XXXIII do art. 5o, no inciso II do $\S 3^{\circ}$ do art. 37 e no $\S 2^{\circ}$ do art. 216 da Constituição Federal. 
I - os órgãos públicos integrantes da administração direta dos Poderes Executivo, Legislativo, incluindo as Cortes de Contas, e Judiciário e do Ministério Público" .

Pela previsão legal, todas as instituições públicas brasileiras, nas diferentes esferas e Poderes, foram compelidas a implantar as diretrizes da LAI, não só por força da lei, mas por ser uma norma fundamental para o acesso à informação e transparência pública, garantindo o exercício da democracia com mecanismos que permitam aos cidadãos fiscalizar as instituições e o governo.

Já existe garantia constitucional do acesso à informação (art. 5º, XXXIII da CRFB/88) desde 1988. No entanto, somente após 23 anos foi que o Brasil regulamentou esse direito por meio da Lei de Acesso à Informação Pública. É importante observar que o tema já tinha sido contemplado - embora jamais efetivado - no artigo $5^{\circ}$ da Lei No 8.159, de 9 de janeiro de 1991 (Lei de Arquivos).

A LAI descreve quais os procedimentos que se destinam a assegurar o direito fundamental de acesso à informação e que estes devem ser executados em conformidade com os princípios básicos da administração pública, como ilustra o site da Fundação de Amparo à Pesquisa do Estado do Maranhão (Figura 1).

A FAPEAM Fomento à Pesquisa Serviços Downloads Ouvidoria Acesso à Informação Fale ConoscolAtendimento Portal do Servidor

FAPEAM > Transparência

BUSCA Pesquisar..._ Buscar

Transparência

\section{Bem Vindo ao Portal de Acesso à Informação da FAPEAM}

A FAPEAM já disponibiliza inúmeras informações em seus canais digitais. Em cumprimento à Lei de Acesso à Informação (Lei 12.527 /2011), a partir de agora você conta com mais um canal de informações.

Publicar essas informações é uma atitude transparente que a FAPEAM adota seguindo seus principios de governança corporativa. É mais uma demonstração de transparência e responsabilidade da empresa com a sociedade.

Esse Portal contempla as informações da FAPEAM - Fundação de Amparo à Pesquisa do Estado do Amazonas você tem acesso as informações pelos links ao lado.

Caso não encontre a informação desejada poderá solicitá-la através de nosso Serviço de Informação ao Cidadão da FAPEAM.

Responsável pela Execução da Lei de Acesso à Informação: Silvana Suelly Noberto Silva Bezerra Telefone: (92) 3878-4041 - Para esclarecimentos sobre esse canal.

Horário: Segunda a sexta-feira, das $8 \mathrm{~h}$ às $15 \mathrm{~h}$

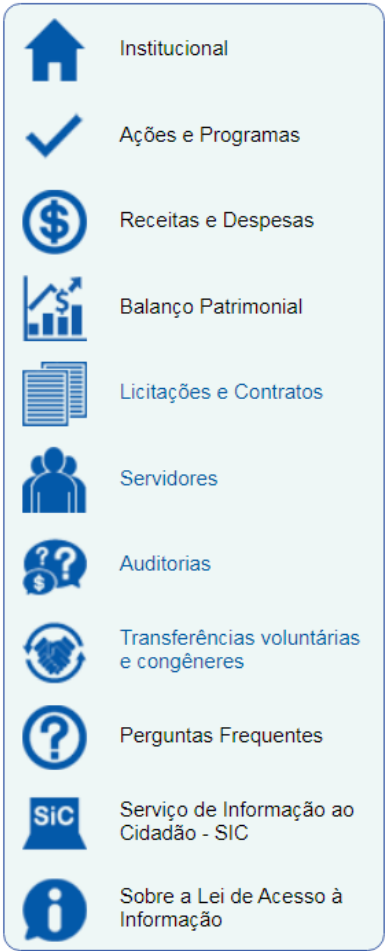

Figura 1 - Transparência das informações da Fundação de Amparo à Pesquisa do Estado do Maranhão (FAPEAM) Fonte: http://www.fapeam.am.gov.br/transparencia/

Apesar de anos de criação da LAI, é registrada a omissão e/ou opacidade de informações pelas agências estatais de fomento à pesquisa, conforme observamos, em janeiro de 2019, nas apresentações de editais do Programa de Pesquisa para o SUS (PPSUS), do Ministério da Saúde, nos sites das Fundações de Amparo à Pesquisa nos diversos estados e no Distrito Federal, no período de interesse do estudo, entre os anos 2009 a 2018, na Seção de editais encerrados e resultados, conforme podemos observar nos registros do Quadro 1. 
Quadro 1-Apresentação sobre o registro de editais PPSUS nas FAPS

\begin{tabular}{|c|c|c|c|c|c|c|c|c|c|c|}
\hline Estado/Ano & 2009 & 2010 & 2011 & 2012 & 2013 & 2014 & 2015 & 2016 & 2017 & 2018 \\
\hline \multicolumn{11}{|l|}{ Região Sul } \\
\hline \multicolumn{11}{|l|}{ RS } \\
\hline \multicolumn{11}{|l|}{ SC } \\
\hline \multicolumn{11}{|l|}{ PR } \\
\hline \multicolumn{11}{|l|}{$\begin{array}{l}\text { Região } \\
\text { Sudeste }\end{array}$} \\
\hline \multicolumn{11}{|l|}{ SP } \\
\hline \multicolumn{11}{|l|}{ RJ } \\
\hline \multicolumn{11}{|l|}{ ES } \\
\hline \multicolumn{11}{|l|}{ MG } \\
\hline \multicolumn{11}{|l|}{$\begin{array}{l}\text { Região } \\
\text { Norte }\end{array}$} \\
\hline \multicolumn{11}{|l|}{ AC } \\
\hline \multicolumn{11}{|l|}{ AM } \\
\hline \multicolumn{11}{|l|}{ AP } \\
\hline \multicolumn{11}{|l|}{ PA } \\
\hline \multicolumn{11}{|l|}{ RO } \\
\hline \multicolumn{11}{|l|}{$\mathbf{R R}$} \\
\hline \multicolumn{11}{|l|}{ TO } \\
\hline \multicolumn{11}{|l|}{$\begin{array}{l}\text { Região } \\
\text { Nordeste }\end{array}$} \\
\hline \multicolumn{11}{|l|}{ AL } \\
\hline \multicolumn{11}{|l|}{ BA } \\
\hline \multicolumn{11}{|l|}{$\mathbf{C E}$} \\
\hline \multicolumn{11}{|l|}{ MA } \\
\hline \multicolumn{11}{|l|}{ PB } \\
\hline \multicolumn{11}{|l|}{ PE } \\
\hline \multicolumn{11}{|l|}{ PI } \\
\hline \multicolumn{11}{|l|}{$\mathbf{R N}$} \\
\hline \multicolumn{11}{|l|}{ Se } \\
\hline $\begin{array}{l}\text { Região } \\
\text { Centro- } \\
\text { Oeste }\end{array}$ & & & & & & & & & & \\
\hline DF & & & & & & & & & & \\
\hline GO & & & & & & & & & & \\
\hline MS & & & & & & & & & & \\
\hline MT & & & & & & & & & & \\
\hline
\end{tabular}

Fonte: Elaborada pelos autores (2019).

Legenda: Registro de editais PPSUS nas FAPs.

Vale apontar que o estado de Roraima, por exemplo, não possui Fundação Estadual de Fomento à Pesquisa e as lacunas de informações de editais nas demais instituições, seja em alguns anos ou na totalidade do período focado no estudo, desencadeiam o debate da comunicação e da informação enquanto lócus, possuidor de conhecimentos para produções de bens e de serviços, que fomentam políticas públicas e concretizam a responsabilidade social das instituições públicas. 
Nesse contexto crítico apresentado, informações imprecisas para apurações de análises de investimentos podem ser geradas no campo de Ciência, Tecnologia e Inovação em sistemas locais e nacional, devido à amplitude deste campo. Assim, ele está a exigir a adoção de estruturas flexíveis de financiamento para a pesquisa, requerendo o estabelecimento de parcerias com outras instituições de fomento no país, especialmente, no âmbito federal para responder às demandas apresentadas.

O Programa de Pesquisa para o SUS (PPSUS) é um exemplo dessa ação técnico-científica articulada e é uma iniciativa de fomento à pesquisa em saúde, instituída pelo Ministério da Saúde, a partir de 2002, tendo com diretriz principal: "apoiar financeiramente o desenvolvimento de pesquisas que visem contribuir para a resolução dos problemas prioritários de saúde da população brasileira e para o fortalecimento da gestão do Sistema Único de Saúde"7.

O PPSUS, por meio de editais, visa ao fortalecimento dos sistemas locais de pesquisa, apoiando o desenvolvimento de projetos com finalidade de melhorias nas demandas dos sistemas e serviços de saúde e desconcentrando o investimento destinado à Ciência, Tecnologia e Inovação em Saúde (C\&TIS), por intermédio de parcerias institucionais, nos âmbitos federal e estaduais. Leva em conta o envolvimento do Conselho Nacional de Desenvolvimento Científico e Tecnológico (CNPq) no gerenciamento administrativo juntamente com as Fundações de Amparo e/ou Apoio à Pesquisa e as Secretarias Estaduais de Saúde, lançando editais de projetos de pesquisa com temas elencados, após consultas públicas a diversas instituições envolvidas no campo de conhecimento, e coadunados com as prioridades estabelecidas na Agenda Nacional de Prioridades de Pesquisa em Saúde (ANPPS), do Ministério da Saúde, de acordo com interesses locais ${ }^{8-10}$.

É significativo reportarmos ao ano de 2000, na ocasião do VI Congresso Brasileiro de Saúde Coletiva, quando o Grupo Técnico de Informação em Saúde e População da Associação Brasileira de Saúde Coletiva (GTISP/Abrasco) foi destacado durante a realização de uma oficina de trabalho com interesse em planejar a construção de uma Política Nacional de Informações em Saúde ${ }^{i}$, visando à melhoria do uso das informações pelos diversos atores sociais envolvidos com o SUS em todo o país. "Desde sua criação, o GTISP/Abrasco vem sugerindo a necessidade de se criarem mecanismos que promovam a melhoria da qualidade das informações. Apesar da melhoria dos Sistemas de Informação em Saúde, verificada nos últimos anos, seu aperfeiçoamento continua e sempre será desejável. É necessário baixar, cada vez mais, a tolerância com as deficiências na produção de informações, desde o registro até a disponibilização" ${ }^{\prime 1}$.

A institucionalização da Política Nacional de Informações e Informática em Saúde foi alcançada pela Portaria $n^{0}$ 589/15, publicada no Diário Oficial da União (DOU), de 22 de maio de 2015. Atende, primordialmente, ao art. 47 da Lei no 8.080/90, que dispõe: "O Ministério da Saúde, em articulação com os níveis estaduais e municipais do Sistema Único de Saúde (SUS), organizará, no prazo de o2(dois) anos, um sistema nacional de informação em saúde, integrado em todo o território nacional, abrangendo questões epidemiológicas e de prestação de serviços"12.

De Souza ${ }^{13}$ nos aponta a complexidade existente na formulação de políticas e na gestão de sistemas utilizando conhecimentos científicos, especialmente, no "decorrer das próprias características do fazer científico e das peculiaridades dos processos de formulação de políticas e de condução de organizações de saúde" ${ }^{13}$, conciliando suas racionalidades nos processos de fazer e da divulgação de dados e atentando para as ressignificações de linguagens que os espaços de comunicações exigem para suas apresentações. "Em suma, para compreender a utilização de conhecimentos científicos na gestão, é importante superar a visão reificadora e conceber a decisão como resultado de processos complexos e limitados, racionais e intuitivos, caóticos e simbólicos que envolvem necessariamente relações de poder"13.

Dessa forma, concebe-se como um jogo de linguagens com a agregação do conhecimento científico as orientações de formulações e de avaliações de políticas realizadas pelos gestores dos sistemas. Os discursos

i Sobre a institucionalização da Política Nacional de Informações em Saúde, consultar a mencionada lei². 
sobre saúde, como apontam Castiel e Álvarez-Dardet Diaz ${ }^{14}$, possuem uma amplitude do campo da saúde, com a significância de “(...) situá-los em determinados momentos históricos e saber as razões por que se legitimam ao acompanharem a ordem econômica, política e social onde são gerados, sustentados e replicados a se ajustarem a ela. É fundamental se destacar que essa observação serve não apenas para o discurso jornalístico, mas também para o discurso científico"14.

Para a eficácia desse processo, é importante o cuidado de se colocar na equação das diversas informações sobre saúde, pautando a democratização na tomada de decisões e a mudança cultural relativa à superação das resistências ao uso das informações e suas tecnologias no processo de gestão da saúde com intuito da formulação de uma política de informações em saúde.

Primordialmente, não podemos negligenciar que, na área de políticas de saúde, o encontro da ciência com o senso comum cria 'um senso comum erudito', desencadeando a formação democrática de conhecimentos e de gestão dos sistemas de saúde com a pluralidade de sistemas de pesquisa e a formulação de políticas com as Instituições mais eficientes, com a participação ampla dos setores sociais na democratização da sociedade ${ }^{15}$.

Analisando o contexto de produção de informação em saúde relacionado às atenções éticas e jurídicas, Moraes e Santos ${ }^{11}$ assinalam o avanço tecnológico associado à informação em saúde: "contribuindo para a criação de melhorias para a gestão da saúde e facilidades para o cidadão, também traz riscos no que se refere à garantia de sua privacidade. O Brasil pouco avançou em relação à criação de salvaguardas éticas e jurídicas que garantam a segurança da inviolabilidade da identificação do cidadão em qualquer base de dados existente" ${ }^{\prime 11}$.

Há uma crescente atenção internacional voltada para informações da pesquisa científica no processo de formulação de políticas de saúde ${ }^{16,17}$. No entanto, esse complexo fenômeno social exige o envolvimento de várias instituições governamentais e não governamentais e vários atores sociais

O relatório preparado pela Organização Mundial da Saúde ${ }^{18}$ aborda as desigualdades no processo de pesquisa, registrando que a maioria dos países em desenvolvimento tem escassez de pesquisadores ou institutos de pesquisa adequadamente equipados, sem adaptação local e adequação dos esforços de pesquisa realizados em outras regiões. Pelo contrário, esse documento enfatiza que a pesquisa em saúde nos países em desenvolvimento é muitas vezes realizada em parceria com outros países e seus resultados estão desconectados das realidades locais, assim como registra o primado da pesquisa biomédica e clínica em pesquisa sobre determinantes sociais de pesquisa em saúde e seus sistemas.

Ao analisar a estrutura de desenvolvimento tecnológico dos países subdesenvolvidos, Herrera ${ }^{19}$ ressalta que um dos principais determinantes do atraso científico e tecnológico é o chamado efeito institucional. Esses países têm uma política científica com seus próprios objetivos e sua resistência.

Assim, esse autor enfatiza a distinção entre política científica explícita, expressa por lei, regulamentos e estatutos e política científica implícita, caracterizada pela demanda científica e tecnológica, a qual constitui a verdadeira política científica no trabalho, sem necessariamente haver contradições entre elas.

A ideia de política baseada em evidências foi apropriada pelo estado moderno e na saúde recebeu grande atenção. De acordo com Barreto $^{20}$, esse procedimento aumentará as possibilidades de tomar decisões mais efetivas, que resultam em benefícios como a redução de custos para a população em geral.

Diante do exposto, a finalidade primordial deste artigo é identificar nos editais do Programa de Pesquisa para o SUS, registrados nos sites de Fundações Públicas de Amparos à Pesquisa, ações voltadas para projetos de pesquisa sobre a saúde da população de lésbicas, gays, bissexuais e transgêneros (LGBT), em razão da complexidade no contexto do adoecimento desse grupo, que merece atenções do SUS para formulações de políticas específicas, incluindo o incentivo de estudos em diversas áreas de conhecimento.

Dessa forma, o processo de construção e consolidação de políticas deve ser compreendido em sua dinamicidade, envolvendo diversos atores sociais e atividades institucionais. Nessa dinâmica, debater 
gênero e saúde é apontar respostas com base no reconhecimento da existência de processos de discriminação e de exclusão relativa à saúde da população LGBT.

Vale ressaltar que os movimentos sociais organizados, ainda na década de 1980, pressionaram o governo federal e conquistaram uma política de assistência especializada para o combate ao HIV/Aids no país, que dura até os dias atuais como política pública, demonstrando, talvez pela primeira vez, uma preocupação do Estado brasileiro com a saúde das pessoas LGBT ${ }^{21,22}$.

A Política Nacional de Saúde Integral de Lésbicas, Gays, Bissexuais, Travestis e Transexuais busca consolidar-se como resposta do Estado brasileiro frente às necessidades particulares no âmbito do SUS e traz avanço na política de saúde ao apontar, como uma de suas diretrizes, que a população LGBT é portadora de direitos humanos, especialmente diante de um quadro de exclusão histórica nos diversos setores sociais. "Ampliar o acesso de lésbicas, gays, bissexuais, travestis e transexuais aos serviços de saúde do SUS, garantindo o respeito às pessoas e acolhimento com qualidade e resolução de suas demandas e necessidades”23.

A concepção de um edital do Programa de Pesquisa para o SUS (PPSUS), conforme abordamos anteriormente, é alicerçada através da Agenda Nacional de Prioridades de Pesquisa em Saúde, implementada em 2008, sendo "[...] um processo político que busca, em todas as suas etapas, a ampla participação de atores com experiências e linguagens distintas tanto da pesquisa como da saúde. A articulação em torno da Agenda é a ação mais importante na legitimação deste instrumento na Política Nacional de Ciência, Tecnologia e Inovação em Saúde no País, e permitirá que prioridades de pesquisa em saúde estejam em consonância com os princípios do SUS”24.

Ao observar o Sumário dessa Agenda, registramos a ausência de tema de pesquisa sobre população LGBT por ela ser vulnerável quanto ao atendimento de seus direitos humanos, incluindo o acesso aos serviços públicos de saúde. No entanto, executar projetos de pesquisa com esta população no âmbito do Programa em questão atende à equidade em saúde, identificando a oportunidade de disseminações de informações em sites institucionais para fins de análises históricas.

\section{SUMÁRIO}

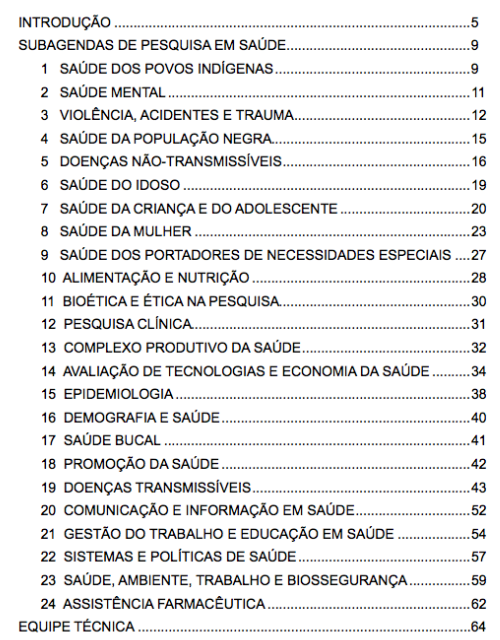

Figura 2 - Sumário da Agenda Nacional de Prioridades de Pesquisa em Saúde Fonte: Ministério da Saúde ${ }^{25}$. 
Conforme apontado anteriormente, como método para o estudo, foi realizado um levantamento de editais publicados e de projetos de pesquisa contemplados no PPSUS, voltados para a instituição da Política Nacional de Saúde Integral de Lésbicas, Gays, Bissexuais, Travestis e Transexuais, que visam contemplar a população em foco em sites institucionais do Ministério da Saúde e das Fundações Estaduais de Amparo à Pesquisa.

Delineando um quadro global de produção científica sobre pessoas transgêneras na Web of Science (WOS), espaço interdisciplinar, Rodrigues, Silva e Araújo ${ }^{26}$ identificaram 8.839 estudos em diversas áreas de conhecimento, registrando os Estados Unidos como o país de maior número de trabalhos produzidos (46,68\%), seguido por dois outros países de língua inglesa: a Inglaterra (7,39\%) e o Canadá (7,34\%); o Brasil ocupa a $11^{\text {a }}$ posição $(1,76 \%)$. As autoras do mencionado trabalho apontam que as conquistas de direitos políticos da população LGBT e os espaços midiáticos de visibilidades de celebridades contribuem para o crescimento de interesse desse campo de estudo.

A internet tornou-se, contemporaneamente, uma ferramenta interessante também no processo de pesquisa acadêmica e não acadêmica devido à sua amplitude em relação às informações disseminadas e às oportunizações de coletas e de análises de dados em diversos canais de acessos, produzidos por instituições governamentais, não governamentais e indivíduos, com interesses diversos.

Sem restrições de espaço e de tempo, o pesquisador, obviamente com tecnologias apropriadas, acessa canais de armazenamentos de informações, com interfaces de linguagens diversas, que tornarão rica sua atividade de investigação, otimizando o retorno dos métodos de coleta de dados e a economicidade nos processos dessa atividade visto que os custos envolvidos em pesquisas tradicionais costumam ser altos. "O baixo custo, a alta velocidade e relativa simplicidade de pesquisa via Internet irão certamente encorajar o crescimento do número de pessoas que irão desejar realizar suas próprias pesquisas diretamente, sem a necessidade de passar por organizações ou grupos que realizam pesquisa" ${ }^{27}$.

Como um dos efeitos desse processo, o uso da internet para fins de pesquisa traz em cena, primordialmente, a atenção voltada para a seletividade de dados em virtude da ampla disponibilidade deles; o questionamento das modalidades de obtenções e de análises de dados pelos atores sociais envolvidos nessa atividade, redimensionando os papéis sociais atribuídos no seu contexto que impactam a ética da pesquisa. Tudo isso como debatido por Kozinets ${ }^{28}$ sobre o caráter público ou privado e a legitimidades das fontes das informações apresentadas e suas divulgações, subordinadas às questões legais. E mais: mantendo, conforme o objeto da pesquisa, a confidencialidade e o anonimato dos participantes, considerando a amplitude das potencialidades do método etnográfico no campo digital mediante esta proposta metodológica.

A próxima figura apresenta nos sites das Fundações Estaduais de Amparo à Pesquisa e do Distrito Federal o registro da linha de pesquisa sobre saúde da população LGBT nos editais do PPSUS. 
Quadro 2 - Presença da linha de pesquisa sobre saúde da população LGBT nos editais do PPSUS (2009-2018)

\begin{tabular}{|c|c|c|c|c|c|c|c|c|c|c|}
\hline Estado/Ano & 2009 & 2010 & 2011 & 2012 & 2013 & 2014 & 2015 & 2016 & 2017 & 2018 \\
\hline \multicolumn{11}{|l|}{ Região Sul } \\
\hline \multicolumn{11}{|l|}{ RS } \\
\hline \multicolumn{11}{|l|}{ SC } \\
\hline \multicolumn{11}{|l|}{ PR } \\
\hline \multicolumn{11}{|l|}{$\begin{array}{l}\text { Região } \\
\text { Sudeste }\end{array}$} \\
\hline \multicolumn{11}{|l|}{ SP } \\
\hline \multicolumn{11}{|l|}{ RJ } \\
\hline \multicolumn{11}{|l|}{ ES } \\
\hline \multicolumn{11}{|l|}{ MG } \\
\hline \multicolumn{11}{|l|}{$\begin{array}{l}\text { Região } \\
\text { Norte }\end{array}$} \\
\hline \multicolumn{11}{|l|}{ AC } \\
\hline \multicolumn{11}{|l|}{ AM } \\
\hline \multicolumn{11}{|l|}{ AP PPSUS } \\
\hline \multicolumn{11}{|l|}{ PA } \\
\hline \multicolumn{11}{|l|}{ RO } \\
\hline \multicolumn{11}{|l|}{$\begin{array}{l}\text { RR sem } \\
\text { informação }\end{array}$} \\
\hline \multicolumn{11}{|l|}{$\begin{array}{l}\text { TO sem } \\
\text { informação }\end{array}$} \\
\hline \multicolumn{11}{|l|}{$\begin{array}{l}\text { Região } \\
\text { Nordeste }\end{array}$} \\
\hline \multicolumn{11}{|l|}{ AL } \\
\hline \multicolumn{11}{|l|}{ BA } \\
\hline \multicolumn{11}{|l|}{ CE } \\
\hline \multicolumn{11}{|l|}{$\begin{array}{l}\text { MA (sem } \\
\text { informação) }\end{array}$} \\
\hline \multicolumn{11}{|l|}{ PB } \\
\hline \multicolumn{11}{|l|}{ PE } \\
\hline \multicolumn{11}{|l|}{ PI } \\
\hline \multicolumn{11}{|l|}{ RN } \\
\hline \multicolumn{11}{|l|}{$\begin{array}{l}\text { Se (sem } \\
\text { informação) }\end{array}$} \\
\hline $\begin{array}{l}\text { Região } \\
\text { Centro- } \\
\text { Oeste } \\
\end{array}$ & & & & & & & & & & \\
\hline DF & & & & & & & & & & \\
\hline GO & & & & & & & & & & \\
\hline $\begin{array}{l}\text { MS através } \\
\text { de relatórios }\end{array}$ & & & & & & & & & & \\
\hline MT & & & & & & & & & & \\
\hline
\end{tabular}

Fonte: Elaborada pelos autores (2019).

Legenda: $\quad$ Registro de editais PPSUS nas FAPs. 
Considerando a relevância dessa política, constatamos o número baixo de oportunidades de contemplações de projetos de pesquisa nos editais apresentados pelas instituições: somente em quatro edições, é possível encontrar linha de pesquisa específica.

Contudo, ao observar o site Pesquisa Saúde ${ }^{i i}$, espaço virtual agregador de pesquisas científicas com recursos da Secretaria de Ciência, Tecnologia e Insumos Estratégicos do Ministério da Saúde (SCTIE/MS), o sistema apresenta resultados de projetos relacionados com saúde da população em foco em diversas linhas de pesquisas com a palavra-chave LGBT ${ }^{\mathrm{iii}}$ :

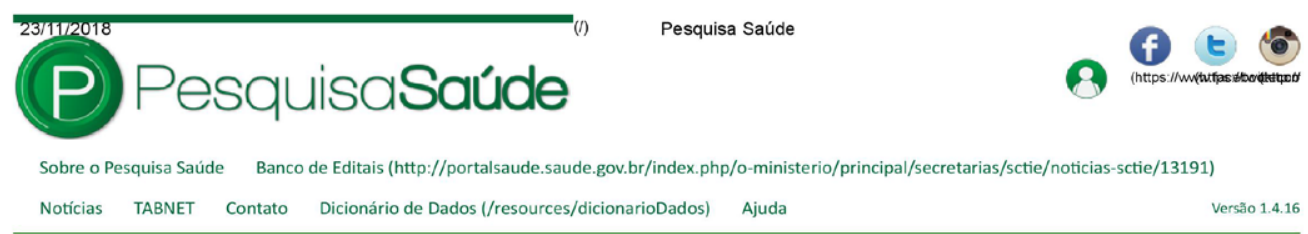

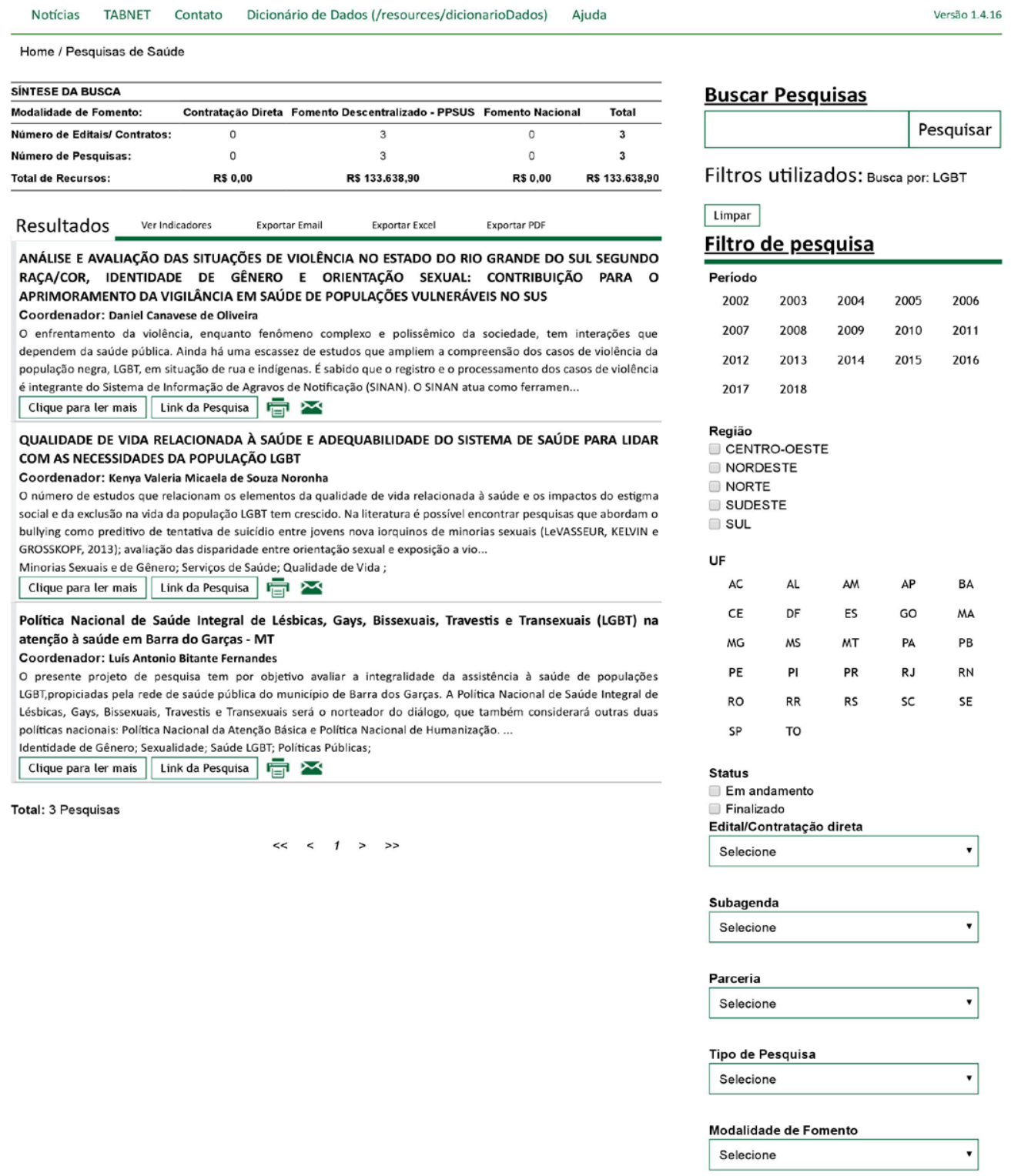

Figura 3 - Pesquisas sobre saúde da população LGBT no site Pesquisa Saúde

Fonte: Sistema Pesquisa Saude ([201?]) $)^{29}$.

Ao detalhar as informações desses projetos contemplados no site Pesquisa Saúde, verificamos que suas concessões ocorreram em distintas linhas de pesquisa, como visto no Quadro 3.

ii Disponível em: http://pesquisasaude.saude.gov.br/

iii Disponível em: http://pesquisasaude.saude.gov.br/pesquisas.xhtml 
Quadro 3 - Projetos Aprovados no PPSUS - Outras Linhas de Pesquisa

\begin{tabular}{|l|l|l|l|}
\hline Estado & Título & Ano & Linha de Pesquisa \\
\hline RS & $\begin{array}{l}\text { Análise e avaliação das situações de violência no estado do } \\
\text { Rio Grande do Sul segundo raça/cor, identidade de gênero } \\
\text { e orientação sexual: contribuição para o aprimoramento da } \\
\text { vigilância em saúde de populações vulneráveis no SUS }\end{array}$ & 2017 & $\begin{array}{l}\text { Violência, acidentes e } \\
\text { trauma }\end{array}$ \\
\hline MG & $\begin{array}{l}\text { Qualidade de vida relacionada à saúde e adequabilidade do } \\
\text { sistema de saúde para lidar com as necessidades da população } \\
\text { LGBT }\end{array}$ & 2017 & Demografia e saúde \\
\hline MT & $\begin{array}{l}\text { Política Nacional de Saúde Integral de Lésbicas, Gays, } \\
\text { Bissexuais, Travestis e Transexuais (LGBT) na atenção à saúde } \\
\text { em Barra do Garças - MT }\end{array}$ & 2017 & Promoção da saúde \\
\hline
\end{tabular}

Fonte: Elaborada pelos autores (2019).

Também observando nesse site a totalidade de fomento de 2.454 projetos contemplados desde o início do PPSUS, emerge a apreensão de observar se efetivamente a saúde da população LGBT é um tema de interesse de estudos e de prioridade de pesquisa no atual cenário de Ciência, Tecnologia, Inovação e Saúde.

\section{Considerações finais}

A falta de políticas públicas de informação e de respeito aos princípios constitucionais atinentes ao acesso à informação, a partir da interpretação dos direitos fundamentais, cria uma atmosfera de desorganização da informação e, consequentemente, contribui para a cultura do sigilo que persiste na sociedade brasileira.

É dever da Administração Pública a produção e a coleta de informações, seja por meio da elaboração de mapas que significam o registro do território de domínio, seja por realização de censos e estatísticas com os registros da população, visando, primordialmente, ao cidadão no que tange a algumas orientações no seu cotidiano.

Conforme ilustrado neste artigo, pode-se apreender que os sites das Fundações citadas apresentam um hiato entre a tecnologia da informação e comunicação no que se refere à sua funcionalidade como ferramentas governamentais virtuais. Esta lacuna inviabiliza a incrementação da intimidade com o Estado e a democratização da informação para o cidadão e, por consequência, sua autonomia na busca de conhecimentos e de possíveis formulações de políticas.

Cabe observar a institucionalização da Política Nacional de Informações em Saúde em consonância com a Lei $\mathrm{n}^{\circ}$ 8.080/90 objetivando a melhoria da qualidade de produção de informações. Assim, permite que os resultados de projetos de pesquisa fomentados pelo Programa de Pesquisa para o SUS sejam disseminados em sites institucionais envolvidos nas suas operacionalizações e ratifica a consolidação dos sistemas estaduais de ciência, tecnologia, inovação e saúde. Trata-se de um incentivo ao envolvimento de diversas instituições interessadas na produção de conhecimento junto ao SUS, assim como na incrementação da viabilidade das bases científico-tecnológicas de produtos e processos de saúde.

O desenvolvimento do PPSUS oportuniza articulações políticas do campo de Ciência, Tecnologia e Inovação em Saúde nas esferas federal e estaduais com mobilizações de diversos setores envolvidos em pesquisas relacionadas à saúde. Desta maneira, ratifica a descentralização das ações, sobretudo na definição das prioridades dessa agenda de pesquisa em saúde, registrando a ausência de expressão do campo da saúde da população LGBT no âmbito da Agenda Nacional.

Ao observar, nos sites mencionados, registros de projetos de pesquisa sobre saúde da população LGBT, valorizou-se a dimensão da usabilidade, apreendendo desorganização nas apresentações dos editais e dos resultados, o que proporciona ineficiência na sua operacionalização e na funcionalidade das formulações de 
políticas públicas do campo da saúde. Já o site Pesquisa Saúde, do Ministério da Saúde, enquanto lócus de informações do Programa de Pesquisa para o SUS, mostra-se eficiente para monitorar estudos sobre saúde da população LGBT.

Cabe ressaltar que o estudo não teve a pretensão de esgotar os estudos sobre a LAI e seus desdobramentos nas Fundações de Amparo à Pesquisa; ao contrário, deve ponderar a inovação na metodologia e no arranjo dos argumentos.

Por fim, apontamos a necessidade de se realizar um aumento da qualidade de informações nos sites focados, visando a uma melhoria na tomada de decisões no campo de Ciência, Tecnologia e Inovação e incentivos a fomentos, especificamente, de estudos para fortalecimento do campo da saúde da população LGBT, visto que a atual conjuntura caracteriza-se como ofensa ao princípio da cidadania plena e provoca prejuízo ao desenvolvimento da política de saúde, facilitando assim a permanência dos padrões ideológicos dominantes, além de inibir novas proposições de mudanças de paradigmas.

\section{Referências}

1. Wanderley MB. Sistema de informação em gestão social. Estud. Av. [Internet]. 2006 [citado 2019 ago 27];20(56):149-58. Disponível em: http://www.scielo.br/scielo.php?script=sci arttext\&pid $=$ S0103-40142006000100011.

2. Vilella RM. Conteúdo, usabilidade e funcionalidade: três dimensões para avaliação de portais estaduais de governo eletrônico na web 2 [dissertação]. Belo Horizonte: Universidade Federal de Minas Gerais; 2003.

3. Centro Latinoamericano de Administracion para el Desarrollo (VE). La responsabilización en la Nueva Gestión Pública Latinoamericana. Buenos Aires: CLAD, BID, Eudeba; 2000.

4. Barbosa AF et al. Governo Eletrônico: um modelo de referência para sua implementação. Anais do Congresso Anual de Tecnologia - CATI; 2004 jun. 22-25; São Paulo: FGV-EAESP; 2004.

5. Presidência da República (BR). Casa Civil. Subchefia de Assuntos Jurídicos. Constituição da República Federativa do Brasil de 1988. Brasília: Congresso Nacional; 1988.

6. Presidência da República (BR). Casa Civil. Subchefia de Assuntos Jurídicos. Lei no 12.527, 18 de novembro de 2011. Regula o acesso a informações previsto no inciso XXXIII do art. $5^{\circ}$, no inciso II do $\S 3^{\circ}$ do art. 37 e no $\S 2^{\circ}$ do art. 216 da Constituição Federal; altera a Lei no 8.112, de 11 de dezembro de 1990; revoga a Lei no 11.111, de 5 de maio de 2005, e dispositivos da Lei no 8.159, de 8 de janeiro de 1991; e dá outras providências.DOU [Internet]. 2011 [citado 2019 ago 27]. Disponivel em: http:// www.planalto.gov.br/ccivil 03/ ato2011-2014/2011/lei//12527.htm.

7. Ministério da Saúde (BR). Secretaria de Ciência, Tecnologia e Insumos Estratégicos. Departamento de Ciência e Tecnologia. Programa de Pesquisa para o SUS: gestão compartilhada em saúde: diretrizes técnicas [Internet]. 5. ed. Brasília: Ministério da Saúde; 2014 [citado 2019 ago 27]. Disponível em: http://u.saude.gov.br/images/pdf/ 2015/marco/30/diretrizes-tecnicas-PPSUS-2014.pdf.

8. Peters LR. O Programa Pesquisa Para o SUS: gestão compartilhada em saúde - PPSUS como ferramenta de descentralização do fomento à pesquisa em saúde [tese]. São Paulo: Universidade de São Paulo; 2013.

9. Oliveira MM. Programa pesquisa para o SUS: gestão compartilhada em saúde - PPSUS: construção do modelo lógico e da matriz de medidas avaliativas [dissertação]. Brasília: Universidade de Brasília; 2008.

10. Guimarães R. Bases para uma política nacional de ciência, tecnologia e inovação em saúde. Ciênc saúde coletiva. 2004;9(2):375-88.

11. Moraes IHS; Santos SRFR. Informações para a gestão do SUS: necessidades e perspectivas. Inf. Epidemiol. Sus [Internet]. 2001 [citado 2019 ago 27];10(1):49-56. Disponível em: http://scielo.iec.gov. br/scielo.php?script= sci arttext\&pid=S0104-16732001000100006\&lng=pt\&nrm=iso.

12. Ministério da Saúde (BR). Secretaria-Executiva. Departamento de Monitoramento e Avaliação do SUS. Política Nacional de Informação e Informática em Saúde. Brasília: Ministério da Saúde; 2016.

13. Souza LEPF. A utilização do conhecimento científico na gestão da saúde. In: Mandarino ACS, Gallo E, Gomberg E, organizadores. Informar e educar em saúde: análises e experiências. Salvador, Rio de Janeiro: Edufba/Editora Fiocruz; 2014. p. 93-106. 
14. Castiel LD, Diaz CAD. A saúde persecutória: os limites da responsabilidade. Rio de Janeiro: Editora Fiocruz; 2007.

15. Santos BS. Introdução a uma ciência pós-moderna. Rio de Janeiro: Graal; 1989.

16. World Health Organization (WHO). Comittee on Health Research: a research agenda for science and technology to support global health development: collaborating venture for global modeling of health. Washington: WHO; 1998.

17. World Health Organization (WHO). Ad hoc Committee on Health Research relating to future intervention options: investing in health research and development. Washington: WHO; 1996.

18. WHO. Preventing chronic diseases: a vital investment. Genebra: World Health Organization; WHO; 2005.

19. Herrera AO. Los determinantes sociales de la política científica en América Latina: Política Científica Explícita y Política Científica Implícita. Desarrollo Económico. 1973;13(49):113-34.

20. Barreto ML. O conhecimento científico e tecnológico como evidência para políticas e atividades regulatórias em saúde. Ciênc. saúde coletiva [Internet]. 2004 jun [citado 2019 ago 30];9(2):329-338. doi: http://dx.doi.org/10.1590/S1413-81232004000200010.

21. Ministério da Saúde (BR). Secretaria de Gestão Estratégica e Participativa. Departamento de Apoio à Gestão Participativa. Transexualidade e travestilidade na saúde. Brasília: Ministério da Saúde; 2015.

22. Lionço T. Atenção integral à saúde e a diversidade sexual no processo transexualizador do SUS: avanços, impasses, desafios. Physis. 2009;19(1):43-63.

23. Ministério da Saúde (BR). Secretaria de Gestão Estratégica e Participativa. Departamento de Apoio à Gestão Participativa. Política Nacional de Saúde Integral de Lésbicas, Gays, Bissexuais, Travestis e Transexuais. Brasília: Ministério da Saúde; 2013.

24. Ministério da Saúde (BR). Secretaria de Ciência, Tecnologia e Insumos Estratégicos. Departamento de Ciência e Tecnologia. Agenda nacional de prioridades de pesquisa em saúde. Brasília: Ministério da Saúde; 2008.

25. Ministério da Saúde (BR). Secretaria de Ciência, Tecnologia e Insumos Estratégicos. Departamento de Ciência e Tecnologia. Agenda nacional de prioridades de pesquisa em saúde [Internet]. 2. ed. Brasília: Ministério da Saúde; 2015. Disponivel em: http://brasil.evipnet.org/wp-content/uploads/2017/07/ANPPS.pdf.

26. Rodrigues EG, Silva CH, Araújo IS. Gênero e produção científica: um panorama sobre pessoas transgêneras. In: Anais do $18^{\circ}$ Encontro Nacional de Pesquisa em Ciência da Informação, 2017 out. 2327; Marília: Unesp; Ancib, 2017.

27. Freitas HMR, Janissek-Muniz R, Moscarola J. Uso da internet no processo de pesquisa e análise de dados [Internet]. 2004 [citado em 19 jan. 2019]. Disponível em: http://gianti.ea.ufrgs.br/files/ artigos/2004/2004 147 ANEP.pdf.

28. Kozinets R. The field behind the screen: using netnography for marketing research in online communities. J Mark Res [Internet]. 2002 [cited 2019 ago 30]. Avaiable from: http://research.bus.wisc. edu/rkozinets/printouts/kozinetsFieldBehind.pdf.

29. Ministério da Saúde (BR). Departamento de Ciência, Tecnologia e Insumos Estratégicos. Sistema PesquisaSaúde [Internet]. [201?] [citado em 19 jan. 2019]. Disponível em: http://pesquisasaude.saude. gov.br/pesquisas.xhtml. 